\title{
Multi-site, multi-year monitoring of the oscillating Algol-type eclipsing binary CT Herculis ${ }^{\star} \star \star$
}

\author{
P. Lampens ${ }^{1}$, A. Strigachev ${ }^{2}$, S.-L. Kim³ ${ }^{3}$, E. Rodríguez ${ }^{4}$, M. J. López-González ${ }^{4}$, J. Vidal-Saínz ${ }^{5}$, D. Mkrtichian,7,8, \\ J.-R. Koo ${ }^{3,9}$, Y. B. Kang ${ }^{3,9}$, P. Van Cauteren ${ }^{10,11}$, P. Wils ${ }^{11}$, Z. Kraicheva ${ }^{2}$, D. Dimitrov ${ }^{2}$, J. Southworth ${ }^{12}$, \\ E. García Melendo 5 , and J. M. Gómez Forellad ${ }^{5}$
}

1 Koninklijke Sterrenwacht van België, Ringlaan 3, 1180 Brussel, Belgium e-mail: Patricia.Lampens@oma. be

2 Institute of Astronomy and National Astronomical Observatory, Bulgarian Academy of Sciences, 72 Tsarigradsko Shosse Blvd., 1784 Sofia, Bulgaria

3 Korea Astronomy and Space Science Institute (KASI), Daejeon 305-348, Korea

4 Instituto de Astrofísica de Andalucía, CSIC, PO Box 3034, 18080 Granada, Spain

5 Grup d'Estudis Astronomics, Apdo. 9481, 08080 Barcelona, Spain

${ }^{6}$ Astrophysical Research Center for the Structure and Evolution of the Cosmos, Sejong University, Seoul, Korea

7 Astronomical Observatory, Odessa National University, 650014 Odessa, Ukraine

${ }^{8}$ Crimean Astrophysical Observatory, Nauchny, 98409 Crimea, Ukraine

9 Department of Astronomy and Space Science, Chungnam National University, 305-764 Daejeon, Korea

${ }^{10}$ Beersel Hills Observatory (BHO), Beersel, Belgium

11 Vereniging Voor Sterrenkunde (VVS), Oostmeers 122 C, 8000 Brugge, Belgium

12 Astrophysics Group, Keele University, Newcastle-Under-Lyme ST5 5BG, UK

Received 4 April 2011 / Accepted 4 August 2011

\begin{abstract}
We present the results of a multi-site photometric campaign carried out in 2004-2008 for the Algol-type eclipsing binary system CT Her, the primary component of which displays $\delta$ Scuti-type oscillations. Our data consist of differential light curves collected in the filters $B$ and $V$, which were analysed using the method of Wilson-Devinney (Рновве). After identifying an adequate binary model and removing the best-fit light-curve solution, we performed a Fourier analysis of the residual $B$ and $V$ light curves to investigate the pulsational behaviour. We confirm the presence of rapid pulsations with a main period of $27.2 \mathrm{~min}$. Up to eight significant frequencies with semi-amplitudes in the range 3 to 1 mmag were detected, all of which lie in the frequency range $43.5-53.5 \mathrm{~d}^{-1}$. This result is independent of the choice of the primary's effective temperature $(8200$ or $8700 \mathrm{~K})$ since the light-curve models of the binary are very similar in both cases. This is yet another case of a complex frequency spectrum observed for an accreting $\delta$ Scuti-type star (after Y Cam). In addition, we demonstrate that the amplitudes of several pulsation frequencies provide evidence of variability on timescales as short as 1-2 years, perhaps even less. Moreover, our analysis takes into account some recently acquired spectra, from which we obtained the corresponding radial velocities for the years 2007-2009. Investigation of the O-C diagram shows that further monitoring of the epochs of eclipse minima of CT Her will cast new light on the evolution of its orbital period.
\end{abstract}

Key words. binaries: eclipsing - stars: oscillations - stars: fundamental parameters - stars: individual: CT Her

\section{Introduction}

Asteroseismology aims to understand the pulsation physics of all kinds of stars in order to probe their interiors. Among the classical pulsators, $\delta$ Scuti stars are rather common and are located at the intersection of the Cepheid instability strip and the main sequence. They pulsate primarily in low overtone radial and nonradial acoustic modes with periods of between $30 \mathrm{~min}$ and $6 \mathrm{~h}$. Some may also pulsate in gravity modes (Kurtz 2000). Their excitation mechanism, i.e. the $\kappa$-mechanism active in the partial

* Based on photometric data collected at the observatories listed in Table 1 and spectra acquired at the NAO, Bulgaria, and Calar Alto Observatory, Spain. The Skinakas Observatory is a collaborative project of the University of Crete, the Foundation for Research and Technology - Hellas, and the Max-Planck-Institut für Extraterrestrische Physik.

$\star \star$ Full Tables 3, 4, 9 and 12 are only available at the CDS via anonymous ftp to cdsarc.u-strasbg.fr $(130.79 .128 .5)$ or via http://cdsarc.u-strasbg.fr/viz-bin/qcat?]/A+A/534/A111 ionisation zones of He I and He II, is well-understood. However, unknown amplitude-limiting and mode-selection mechanisms also operate in these pulsators. Only a fraction of the theoretically predicted modes are observed, which results in (too) many free parameters in the pulsation models. It is indeed imperative that we collect knowledge of the fundamental stellar properties of the pulsator independently.

One way is to study the pulsating components of binary (multiple) systems in great detail, as binary (multiple) systems with well-characterized components provide additional constraints for a more reliable modelling. The positions in the H-R diagram and therefore the components' evolutionary statuses can be more accurately determined than in the case of single stars, e.g. for those $\delta$ Scuti stars that are at the (very) end of their Hcore burning phase. The complexity of having to deal with additional components does not generally weigh against the scientific return, although observations spread over even longer timescales may be necessary to distinguish both phenomena. 
About seventy percent of all stars of the Solar neighbourhood are members of binary or multiple systems (67\% for G-M stars, Mayor et al. 2001; 75\% for O-B stars, Mason et al. 2001), although this is usually ignored in the study of stellar pulsation. There is, however, strong evidence that duplicity affects the pulsation properties in specific cases (e.g. the eccentric binaries HD 177863, De Cat \& Aerts 2002; and HD 209295, Handler et al. 2002). This is also predicted from a theoretical point-of-view (Witte \& Savonije 1999). It is thus essential to understand the possible link(s) between binarity and pulsation(s) and to observe whether or not - under certain circumstances - the internal structure and the pulsating properties of these stars might be different from those of the single pulsators (Lampens 2006).

Detached and semi-detached eclipsing binaries (EBs) are particularly powerful tools in astrophysics: their accurate observation enables us to derive the fundamental properties (masses, radii, luminosities) of each component. Studies of EBs also provide the component's effective temperatures, as well as the distance if stellar atmosphere models are used (Maceroni 2006).

We present a detailed photometric study of the Algol-type binary CT Her, an eclipsing binary of mag 11-12 and spectral type A3V+[G3IV] with an orbital period of 1.7863748 days (Samus et al. 2009). Radial velocity measurements were also collected, but owing to the faintness of the system, high-quality spectra could not be easily acquired. The binary CT Her belongs to the group of oscillating Algol-type (oEA) stars comprising $\approx 35$ known members. Up to now, only a few members of this group have been investigated thoroughly: RZ Cas has been observed photometrically as well as spectroscopically over almost a decade (Lehmann \& Mkrtichian 2004; Rodríguez et al. 2004; Soydugan et al. 2006; Lehmann \& Mkrtichian 2008), while a very detailed photometric study of Y Cam was recently finished (Rodríguez et al. 2010). Another large spectroscopic observational study targeting TW Dra was carried out (Lehmann et al. 2009; Tkachenko et al. 2010). CT Her's (O-C) diagram is indicative of a slow orbital evolution (Kreiner et al. 2001). The primary component displays oscillations of type $\delta$ Scuti with a (main) pulsation period of $0.46 \mathrm{~h}(\approx 28 \mathrm{~min})$ and a total amplitude of about $0.02 \mathrm{mag}$ (Kim et al. 2004a). Among the currently known oEA stars, it has one of the shortest orbital periods and the highest ratio $P_{\text {orb }} / P_{\text {puls }}$ (about 95).

The oEA stars are former secondaries of evolved, semidetached eclipsing binaries that are (still) undergoing mass transfer and form a recently detected subclass of pulsators close to the main sequence (Mkrtichian et al. 2002, 2004). Searches for new oscillating Algols were performed by Kim et al. (2004b, 2006) and Mkrtichian et al. (2005, 2006). Pigulski \& Michalska (2007) and Michalska \& Pigulski (2008) looked for them in the ASAS-3 and the OGLE-II public databases. A search using the NSVS database was also performed at the Institute of Astronomy of the Bulgarian Academy of Sciences (Dimitrov et al. 2008a,b, 2009a,b). Their general characteristics and pulsational properties were summarized by Mkrtichian et al. (2005). They are, indeed, excellent laboratories for investigating the effects of mass accretion events as well as of tides unto the pulsation properties. Changes in these properties (amplitudes, modes and/or phases) caused by mass accretion episodes have been observed (e.g. in RZ Cas where strong modal amplitude variations followed an abrupt change in its orbital period; Rodríguez et al. 2004; Mkrtichian et al. 2005). Their pulsational frequencies could be tidally split (as in KW Aur, Fitch 1976) and/or there may be some coupling between the pulsation and the orbital frequency owing to some resonance mechanism (Mkrtichian et al. 2005). Their very different evolutionary history is a challenge for stellar evolution modelling. For example, to reproduce the gainers of RZ Cas, $\mathrm{KO}$ Aql, and S Equ through conservative binary evolution, large initial mass ratios (typically $>3$ ) would be necessary. However, the corresponding high mass-loss rates at the beginning of the Roche lobe overflow stage result in radii larger than the Roche radii. Hence, a non-conservative approach is needed, in which processes are considered that enhance the period without losing too much mass (De Grève et al. 2009). Such studies have not yet been attempted. Detailed and multi-year studies of more oEA stars are essential in order to provide solid grounds for their (future) asteroseismic modelling.

Section 2 deals with the observational campaigns. We present the time series of photometric data in Sect. 3. In Sect. 4, we report on the light-curve modelling. We present the results of the frequency analyses performed on the residual data and discuss the variability of the amplitudes in Sect. 5. We then introduce the set of radial velocities (Sect. 6), and consider the effects caused by the possible presence of a third companion (Sect. 7). We report on the evolution of the orbital period based on a new analysis of the $(\mathrm{O}-\mathrm{C})$ diagram in Sect. 8. A summary and relevant conclusions may be found in Sect. 9.

\section{Observations and data reduction}

\subsection{The multi-site campaign}

High-precision light curves of CT Her (GSC 01509-1142, $V=$ $11.347, B-V=0.203$ ) were collected in the framework of a five-year long multi-site campaign set up with the purpose to study its pulsational behaviour. A logbook of the observations is given in Table 1. We observed in a differential mode from late spring until late summer of the years 2004-2008. The original observations that led to its discovery as an oEA star (Kim et al. 2004a) were also included. The following comparison stars were used: $\mathrm{C} 1=$ GSC 01509-1140 $(V=10.951, B-V=$ $1.414), \mathrm{C} 2=$ GSC 01509-1052 $(V=11.405, B-V=0.779)$, $\mathrm{C}^{\prime}{ }^{\prime}=$ GSC 01509-1130 $(V=12.02), \mathrm{C} 3=$ GSC 01509-0901 $(V=12.20, B-V=0.78)$, and $\mathrm{C} 4=$ GSC 01509-1090 $(V=10.75, B-V=0.48)$.

The characteristics of the various CCD cameras are the following:

- At the Skinakas Observatory of the University of Crete, Greece, the camera is a Photometrics $1024 \times 1024$ with a SITe SI003B chip of grade 1 and a pixel size of $24 \mu \mathrm{m}$ corresponding to a scale of $0.5^{\prime \prime}$ on the sky. The field-of-view is $8.5^{\prime} \times 8.5^{\prime}$ (Papadakis et al. 2003).

- At the Sobaeksan Optical Astronomy Observatory of the Korea Astronomy and Space Science Institute (KASI), South Korea, the camera has a $2048 \times 2048$ SITe chip with a pixel size of $24 \mu \mathrm{m}$ corresponding to a scale of $0.60^{\prime \prime}$ on the sky. The field-of-view is $20.5^{\prime} \times 20.5^{\prime}$.

- At the Observatory of Mt. Lemmon, Arizona, operated by KASI, the camera has a $2084 \times 2084$ Kodak chip with a pixel size of $24 \mu \mathrm{m}$ corresponding to a scale of $0.64^{\prime \prime}$ on the sky. The field-of-view is $22.2^{\prime} \times 22.2^{\prime}$ large.

- At the Observatorio de Monegrillo ${ }^{1}$, North of Spain, the camera is a SX Starlight CCD with a Sony ICX027BL chip (cooled to about $-25^{\circ} \mathrm{C}$ ) and a pixel size of $12.7 \mu \times 16.6 \mu$ corresponding to $1.80^{\prime \prime} \times 1.38^{\prime \prime}$. The field-of-view covered a sky region of $11.5^{\prime} \times 7.7^{\prime}$. The reduction was done using a

${ }^{1}$ http://www . astrogea.org/jvidal/index.html 
P. Lampens et al.: The oscillating Algol-type eclipsing binary CT Her

Table 1. Campaigns and instrumentation. Total number of useful observations and hours.

\begin{tabular}{lccccccccc}
\hline \hline Year & Site & Country & Tel. & Period (\& Obs.) & Nights & No. & Hours & Filter & CODE \\
\hline 2004 & Skinakas & Crete & $1.3-\mathrm{m}$ & Jun.-July (AS) & 4 & 573 & 17 & $B$ & SKB1 \\
2004 & Sobaeksan & Korea & $0.61-\mathrm{m}$ & March (SLK) & 3 & 379 & 10 & $B$ & KRB1 \\
2005 & Skinakas & Crete & $1.3-\mathrm{m}$ & May-Aug. (AS) & 6 & 1091 & 29 & $B$ & SKB2 \\
2005 & Mt. Lemmon & Arizona & $1.0-\mathrm{m}$ & Jun.-July (SLK) & 5 & 643 & 21 & $B$ & KRB2 \\
2005 & Monegrillo & Spain & $0.4-\mathrm{m}$ & Jun.-July (JV) & 15 & 1208 & 67 & $V$ & SPV1 \\
2005 & Beersel & Belgium & $0.4-\mathrm{m}$ & Jun.-July (PVC) & 5 & 430 & 6 & $B$ & BHOB \\
2005 & Beersel & Belgium & $0.4-\mathrm{m}$ & Jun.-July (PVC) & 2 & 517 & 6 & $V$ & BHOV \\
2006 & Skinakas & Crete & $1.3-\mathrm{m}$ & Jun.-July (AS) & 9 & 1427 & 35 & $B$ & SKB3 \\
2006 & Sierra Nevada & Spain & $1.5-\mathrm{m}$ & Aug. (ER+MLG) & 9 & 835 & 18 & $B$ & SPB1 \\
2007 & Sierra Nevada & Spain & $1.5-\mathrm{m}$ & Mar.-May(ER+MLG) & 8 & 2158 & 18 & $B$ & SPB2 \\
2007 & Beersel & Belgium & $0.4-\mathrm{m}$ & March (PVC) & 1 & 83 & 3 & $V$ & BHOV \\
2008 & NAO Rozhen & Bulgaria & $2.0-\mathrm{m}$ & June-July (AS) & 2 & 358 & 12 & $B$ & ROZB \\
2008 & Monegrillo & Spain & $0.4-\mathrm{m}$ & June-July (JV) & 3 & 372 & 10 & $V$ & SPV2 \\
\hline $2004-08$ & All & - & - & - & - & 2180 & 86 & $V$ & ALL \\
$2004-08$ & All & - & - & - & - & 7960 & 166 & $B$ & ALL \\
\hline
\end{tabular}

software package called LAIA (Laboratory for Astronomical Image Analysis) developed by Joan A. Cano ${ }^{2}$.

- At the Beersel Hils Observatory, Belgium, the camera is a SBIG ST10XMe with a chip of grade 1 and a pixel size of $6.8 \mu \mathrm{m}$ corresponding to a scale of $1.43^{\prime \prime}$ on the sky (in $2 \times 2$ binning). The field-of-view is $17.5^{\prime} \times 26^{\prime}$ on the sky. The reduction was done using the Mira AP (v.7) package ${ }^{3}$.

- At the Observatorio Sierra Nevada, South of Spain, the camera has a $2 \mathrm{k} \times 2 \mathrm{k}$ chip with a pixel size of $13.5 \mu \mathrm{m}$ corresponding to a scale of $0.23^{\prime \prime}$ on the sky $(2 \times 2$ binning $)$. The field-of-view is $7.92^{\prime} \times 7.92^{\prime}$ large.

- At the National Astronomical Observatory (NAO) Rozhen, Bulgaria, the CCD is a VersArray $1330 \mathrm{~B}$ with a $1340 \times 1300$ E2V CCD36-40 chip of grade 2 and a pixel size of $20 \mu \mathrm{m}$ corresponding to a scale of $0.258^{\prime \prime}$ on the sky. The field-ofview is $5.76^{\prime} \times 5.59^{\prime}$ large.

Photoelectric photometric data on CT Her were also acquired in the four Strömgren passbands uvby at the Observatorio Sierra Nevada between 2008 March, 31 and 2008 April, 13. The comparison stars were $\mathrm{K} 1=\mathrm{HD} 145122(V=6.13), \mathrm{K} 2=\mathrm{HD}$ $146101(V=8.24)$, and K3 = HD $145549(V=8.16))$. The observations were carried out in the sequence Sky, K1, K2, CT Her, AO Ser, K1, K3, CT Her, AO Ser... because both oEA stars are close enough to each other on the sky. Unluckily, the orbital period was not fully covered by the additional observations.

\subsection{Observational technique}

The binary CT Her was observed using the standard (Johnson) filters $B$ (mostly) and $V$ (less frequently). At the Beersel Hills Observatory (BHO), we follow the specifications for the filters of Bessell (1995). We chose C1 to be the principal comparison star that was commonly used at every observatory. We adopted the filter $B$ as the main filter because of its higher signal-to-noise ratio $(S / N)$ and the larger amplitudes of pulsation expected. Typical exposure times for the $B$ filter were set between $15 \mathrm{~s}$ (e.g. Sierra Nevada) and 60 s (e.g. Skinakas). Typical exposures for the $V$ filter were 20-30 s (e.g. BHO and Skinakas). All observers followed the standard calibration procedure: sets of biases and darks were taken regularly during each night and a set

\footnotetext{
2 http://www.astrogea. org/soft/laia/laia.htm

3 The Mira software is a registered trademark of Mirametrics, Inc., http://www .mirametrics.com/index.htm
}

of five to six flat-fields per filter was obtained during evening and/or morning twilights.

As an example, we describe the full reduction procedure used for images collected at the Skinakas Observatory. All the primary reduction steps were performed using standard ESOMIDAS routines. The frames were processed as follows: subtraction of the residual bias pattern using a median master-bias frame, flat-fielding using a median master flat-field frame, and median cosmic-ray cleaning. Since the field is not crowded, the technique of aperture photometry was applied to extract the differential magnitudes. The fixed aperture photometry was performed using DAOPHOT (Stetson 1987). The binary CT Her and the comparison stars $\mathrm{C} 1, \mathrm{C} 2$ ', and $\mathrm{C} 3$ were measured using an aperture size as close as possible to the value providing the highest $S / N$ (Strigachev 2009). The data consist of differential photometry of the variable star in the sense (CT Her-C1) and of the check stars in the sense $\left(\mathrm{C} 2^{\prime}-\mathrm{C} 1\right)$ and $(\mathrm{C} 3-\mathrm{C} 1)$.

We also observed CT Her in the $B V R$ filters during one night together with standard fields (Landolt 1992), performing all-sky photometry up to an airmass of two at the Skinakas Observatory on Aug. 2005, 1. The coordinates, calibrated magnitudes, and colours of the target and comparison stars are listed in Table 2.

\section{Photometric data - time series}

The influence of interstellar and atmospheric extinction may be crucial when constructing light curves of eclipsing binaries (Prša \& Zwitter 2005a). This is generally the case when matching data from different sites and campaigns but it may also be valid when using data from a single site. Observations from different nights and/or sites usually do not match - their mean level may be shifted, and there may also be a correlation with airmass during some nights. In this case, we needed to apply differential corrections associated with the second-order coefficient of the atmospheric extinction $\left(k^{\prime \prime}\right)$ since the colours of the comparison star (C1) and the variable star are not the same (see Table 2). These corrections were mainly needed for the data acquired in the filter $B$ because of the dependence on wavelength and the greater inhomogeneity of the $B$-data sets collected over a longer period and at a larger number of sites equipped differently.

To correct for this influence, we first computed the magnitude of CT Her based on the magnitude of C1 in Table 2 and the differential values (CT Her-C1). We next adopted a preliminary light-curve model based on known and fitted parameters for the $V$ light curve obtained during the 2005 and 2008 runs 
Table 2. Coordinates and standard system calibrated magnitudes with their errors for the target and comparison stars.

\begin{tabular}{llccccccc}
\hline \hline Ident & GSC & RA & Dec & $B$ & $V$ & $R$ & $B-V$ & $V-R$ \\
\hline CT Her & GSC01509-1142 & 162026.57 & +182716.9 & $11.33 \pm 0.03$ & $11.12 \pm 0.03$ & $11.00 \pm 0.03$ & $0.21 \pm 0.04$ & $0.12 \pm 0.04$ \\
C1 $^{a}$ & GSC01509-1140 & 162033.89 & +182718.5 & $12.06 \pm 0.03$ & $10.80 \pm 0.03$ & $10.11 \pm 0.03$ & $1.26 \pm 0.04$ & $0.69 \pm 0.04$ \\
C2 ${ }^{b}$ & GSC01509-1130 & 162018.61 & +182732.6 & $13.41 \pm 0.03$ & $12.40 \pm 0.03$ & $11.88 \pm 0.03$ & $1.01 \pm 0.04$ & $0.52 \pm 0.04$ \\
C3 & GSC01509-0901 & 162043.00 & +183053.2 & $13.18 \pm 0.03$ & $12.67 \pm 0.03$ & $12.38 \pm 0.03$ & $0.51 \pm 0.04$ & $0.29 \pm 0.04$ \\
\hline
\end{tabular}

Notes. ${ }^{(a)} \mathrm{C} 1$ was also used by Kim et al. (2004a). ${ }^{(b)} \mathrm{C} 2$ ' is not the same star as C2 (Kim et al. 2004a).

Table 3. CT Her $B$ light curve.

\begin{tabular}{lccc}
\hline \hline HJD & $B$ & Code & Year \\
\hline 2453954.47391 & 11.460156 & SPB1 & 2006 \\
2453954.47529 & 11.462120 & SPB1 & 2006 \\
2453954.47609 & 11.460165 & SPB1 & 2006 \\
$\ldots$ & & & \\
\hline
\end{tabular}

Notes. First three lines, full table available at the CDS.

Table 4. CT Her $V$ light curve.

\begin{tabular}{lccc}
\hline \hline HJD & $V$ & Code & Year \\
\hline 2453557.38195 & 11.953100 & SPV1 & 2005 \\
2453557.38406 & 11.938400 & SPV1 & 2005 \\
2453557.38617 & 11.921600 & SPV1 & 2005 \\
$\ldots$ & & & \\
\hline
\end{tabular}

Notes. First three lines, full table available at the CDS.

at the Observatory of Monegrillo, Spain (cf. Table 1). This was possible because the $V$ light curve is less dependent on these corrections. In general, a clear dependence of the residuals on magnitude difference with the airmass was found each night, which we modelled using linear regression. The correction involved the zero-point shift caused by interstellar and atmospheric extinction and the slope due to the term associated with the second-order atmospheric extinction. At any given site with the same instrument and filter, this slope was assumed to be constant, and ,where relevant, we applied the correction. We note that the effect is systematically smaller in the $V$-band than in the $B$-band. The resulting differential magnitudes of every night were concatenated per filter to produce the overall corrected $B$ and $V$ data sets. These data are presented in Tables 3 and 4 . The full tables are available at the CDS only. Table 1 also lists the numbers of observations collected at each observatory. In the subsequent analysis, more than 7900 and 2100 data points, respectively in the filters $B$ and $V$, were treated.

\section{Simultaneous modelling of the light curves}

The $B$ - and $V$-data sets were used to plot the respective light curves phased against the orbital period of 1.7863748 days (Samus et al. 2009). Using this ephemeris, we redetermined the (single) epoch of primary eclipse observed in the filter $V$ and obtained the improved value of $\mathrm{HJD}_{0}=2442522.93270 \pm$ 0.00006 days. The phased light curve is almost complete in the filter $V$ (Fig. 1). However, we lack most of the primary minimum in the filter $B$ (Fig. 2). Our current objective is to compute an adequate model from both curves in order to remove the geometric and photometric effects caused by the orbital motion and to extract the smallest possible residuals for a subsequent frequency analysis. Concise reports of previous analyses (Lampens et al. $2008 \mathrm{a}, \mathrm{b})$ did not include all of the above-mentioned data sets. In

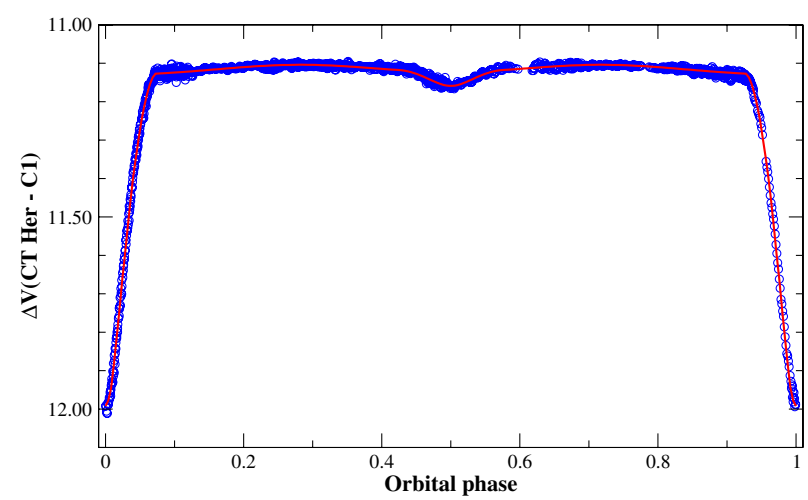

Fig. 1. Observed and theoretical light curve in the filter $V$. Observations are shown as circles. The solid line represents the model.

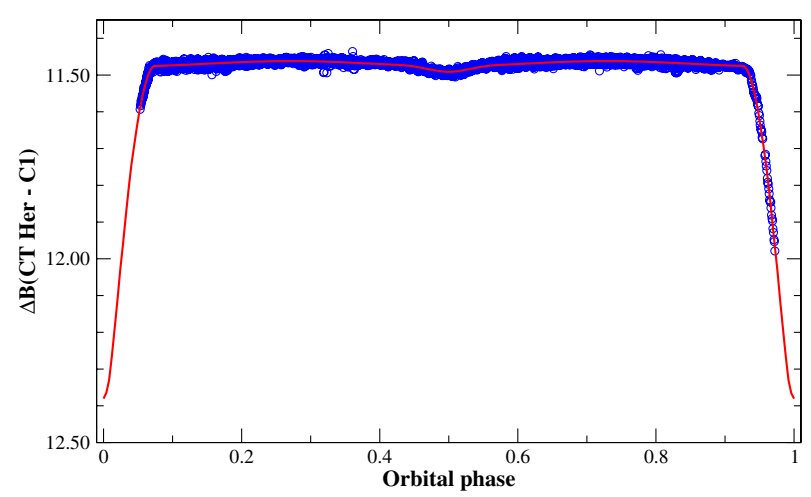

Fig. 2. Observed and theoretical light curve in the filter $B$. Same legend as in Fig. 1.

particular, the full coverage of the primary eclipse had not (yet) been achieved in the filter $V$. The data collected in 2008-2009 were thus used mostly to fill in the gaps in orbital coverage.

We performed a simultaneous modelling of the $B$ and $V$ light curves of CT Her using the light-curve fitting programme Phoebe, version $0.31 \mathrm{a}$, in mode 5, which corresponds to a semidetached binary configuration in which the secondary component fills its limiting lobe, as required for the oEA stars. The package PHozBE ${ }^{4}$ (Prša \& Zwitter 2005b) enables us to compute models of eclipsing binaries based on observed photometric and radial velocity data. It relies on the 2003-version of the widely used Wilson-Devinney code (Wilson \& Devinney 1971; Wilson 1979, 1990). We first employed the almost complete and homogeneous $V$-light curve obtained by one of us (JVS) during the years 2005 and 2008 to compute an initial model; using this initial model, we then corrected the individual $B$ and $V$ data sets (where needed) for the differential effects of atmospheric extinction as explained in Sect. 3. Only then did we compute a best-fit solution for the combined data series.

${ }^{4}$ http://www.fiz.uni-1j.si/phoebe/ 


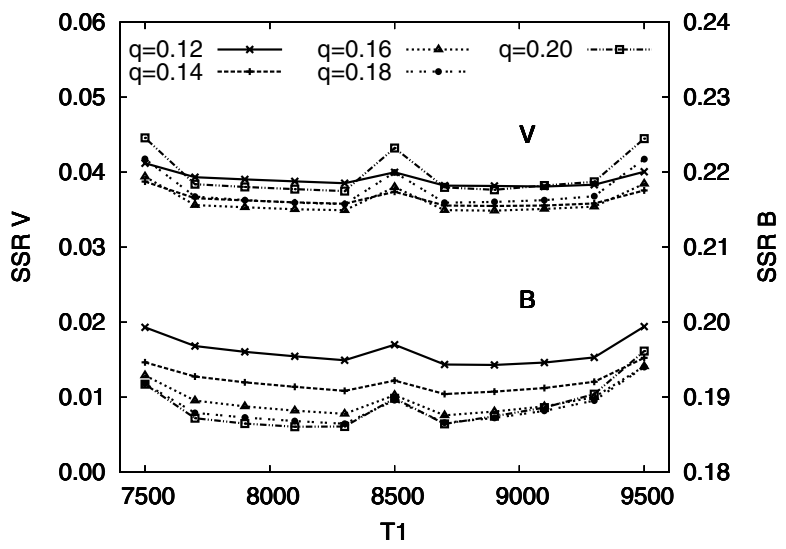

Fig. 3. Distribution of $\chi^{2}$ as a function of $T_{1}$ and $q$.

Since CT Her is classified as an A3V star (Kuznetsova \& Svechnikov 1990), we initially adopted 8700 K as the surface temperature of the primary component. Except for the orbital ephemeris, all other parameters were set as adjustable parameters during the minimization procedure: this concerns the secondary star's surface temperature, $T_{2}$, the inclination, $i$, the mass ratio, $q$, the dimensionless potential, $\Omega_{1}$, and the fractional luminosities of the primary component, $L_{1}$, in $B$ - and $V$-light (six free parameters). We adopted the roughly determined absolute photometric and geometric elements (Svechnikov \& Kuznetsova 1990) as starting values (e.g. $i=82^{\circ}$ for the inclination and $q=0.27$ for the mass ratio), together with an estimate of the secondary component's surface temperature, $T_{2}=5800 \mathrm{~K}$, corresponding to a spectral type of G3IV. Other parameters such as the gravity darkening coefficients $g_{1}$ and $g_{2}$ and the albedos $A_{1}$ and $A_{2}$ were set to the theoretical values corresponding to a radiative atmosphere ( $g_{1}=1.0$ and $\left.A_{1}=1.0\right)$ in the case of the primary component and to a convective atmosphere $\left(g_{2}=0.32\right.$ and $A_{2}=0.5$, Rucínski 1969) in the case of the secondary component. The limb darkening coefficients in the $B$ - and $V$-bands were taken from the tables of Van Hamme (1993). In all the runs, convergence was achieved after two or three iterations only using the differential correction method.

By scanning the distribution of the function to be minimized, $\chi^{2}$, as a function of both $T_{1}$ and the mass ratio, $q$, we found two regions of possible solutions: one region near $T_{1}=8700 \mathrm{~K}$ and another one near $T_{1}=8200 \mathrm{~K}$. Figure 3 illustrates the evolution of $\chi^{2}$ as a function of the parameters $T_{1}$ and $q$. Thus, we considered two equivalent solutions in the subsequent analysis: one solution associated with $T_{1}=8700 \mathrm{~K}$ (model 71) and one associated with $T_{1}=8200 \mathrm{~K}$ (model 72$)$, with their corresponding values of $T_{2}$ and $q$. The latter value of $T_{1}$ corresponds to a spectral type of $\mathrm{A} 5 \mathrm{~V}$ and to a colour index $(B-V)=0.15$ assuming zero reddening (cf. also the observed colour index $(B-V)$ in Table 2). The resulting parameter values and their formal uncertainties are listed in Table 5 . Both models fit the observed $B$ and $V$ light curves very well, as illustrated by the small values of the $\chi^{2}$ function and the $1-2 \%$-level scatters in their residual data sets. The synthetic (and observed) light curves corresponding to the first model are illustrated by Figs. 1 and 2. The synthetic light curves corresponding to the alternative model are indistinguishable.

The main difference between both models is the surface effective temperature of the secondary component, $T_{2}$, which is shifted by about $200 \mathrm{~K}$. The largest changes from the initial values are found in the parameters $T_{2}$ and $q$ : both are smaller than
Table 5. Parameters of the simultaneous light curve solutions for CT Her, including their formal error.

\begin{tabular}{|c|c|c|c|c|}
\hline \multirow[t]{2}{*}{ Parameter } & \multicolumn{2}{|c|}{ Model 71} & \multicolumn{2}{|c|}{ Model 72} \\
\hline & Filter $B$ & Filter $V$ & Filter $B$ & Filter $V$ \\
\hline$i\left(^{\circ}\right)$ & \multicolumn{2}{|c|}{$81.95 \pm 0.01$} & \multicolumn{2}{|c|}{$81.75 \pm 0.01$} \\
\hline & \multirow{2}{*}{\multicolumn{2}{|c|}{$\begin{array}{c}0.1413 \pm 0.0004 \\
8700^{a}\end{array}$}} & \multicolumn{2}{|c|}{$0.1456 \pm 0.0003$} \\
\hline$T_{1}(\mathrm{~K})$ & & & \multicolumn{2}{|c|}{$8200^{a}$} \\
\hline$T_{2}(\mathrm{~K})$ & \multicolumn{2}{|c|}{$4651 \pm 7$} & \multicolumn{2}{|c|}{$4489 \pm 7$} \\
\hline & \multicolumn{2}{|c|}{$4.406 \pm 0.006$} & 4.426 & 0.006 \\
\hline$\Omega_{2}{ }^{c}$ & \multicolumn{2}{|c|}{2.079} & \multicolumn{2}{|c|}{2.091} \\
\hline$\left[L_{1} /\left(L_{1}+L_{2}\right)\right]$ & 0.969 & 0.932 & 0.970 & 0.935 \\
\hline$\left[L_{2} /\left(L_{1}+L_{2}\right)\right]$ & 0.031 & 0.068 & 0.030 & 0.065 \\
\hline$g_{1}$ & \multirow{2}{*}{\multicolumn{2}{|c|}{$1.0^{a}$}} & \multicolumn{2}{|c|}{$1.0^{a}$} \\
\hline$g_{2}$ & \multirow{2}{*}{\multicolumn{2}{|c|}{$\begin{array}{c}0.32^{a} \\
1.0^{a}\end{array}$}} & \multirow{2}{*}{\multicolumn{2}{|c|}{$0.32^{a}$}} \\
\hline$A_{1}$ & & & & \\
\hline$A_{2}$ & \multicolumn{2}{|c|}{$0.5^{a}$} & \multicolumn{2}{|c|}{$0.5^{a}$} \\
\hline$x_{1}{ }^{c}$ & $0.561^{b}$ & $0.476^{b}$ & $0.585^{b}$ & $0.505^{b}$ \\
\hline$x_{2}^{c}$ & $0.934^{b}$ & $0.785^{b}$ & $0.962^{b}$ & $0.811^{b}$ \\
\hline No. data & 7960 & 2180 & 7960 & 2180 \\
\hline$\chi^{2}$ & 0.0160 & 0.0055 & 0.0163 & 0.0056 \\
\hline
\end{tabular}

Notes. ${ }^{(a)}$ Adopted value. ${ }^{(b)}$ Van Hamme (1993) tables. ${ }^{(c)} x_{1}, x_{2}$ are the limb darkening coefficients. $\Omega_{2}$ is the dimensionless potential of the secondary component.

their adopted first guesses (respectively $5800 \mathrm{~K}$ and 0.27 ). The mass ratio is significantly smaller than previously assumed (i.e. $q<0.20)$. Nonetheless, even though the components' effective temperatures are different, both models are obviously similar because their derived physical parameters lie very close to each other (cf. the masses, gravities, and radii of Table 11).

From here on, even though the values of the $\chi^{2}$ function are slightly smaller in the former case (cf. Table 5), we adopt the solution derived with $T_{1}=8200 \mathrm{~K}$ (model 72), the reason being that we obtained a tighter fit with this temperature to a few observed regions of the spectrum of CT Her (Sect. 6). We furthermore show that the frequency analysis of the corresponding residuals is independent of the choice of either one of the two proposed models (Sect. 5).

\section{Frequency analysis of the residual light curves}

The light-curve model with $T_{1}=8200 \mathrm{~K}$ was subsequently subtracted from the original light curves of CT Her to search for short-period pulsations in the residual data. The phased residual light curves are plotted in Figs. 4 and 5, respectively, in the filters $V$ and $B$. The adjustment is excellent for the $B$-data set (without a primary minimum) and fair for the $V$-data set. Weights were associated based on the overall standard deviations of the (CT HerC1) differential $B$-magnitudes computed night by night: while 1.0 was adopted in most cases, some sets with higher noise were allocated a relative weight of 0.2 . This is the case for a few nights at the Beersel Hills Observatory (2005) and two nights at the Observatory of Sierra Nevada (2007). We restricted the residual data to the orbital phase bins in the range $0.05-0.95$ in order to omit the phase of primary minimum (sparsely covered by our V-observations). That various outliers were found close to a primary minimum indicates that it is hard to model both effects simultaneously during this phase of rapid and steep light variation. On the other hand, the outliers are only associated with partially observed eclipses, whereas the fully observed primary 


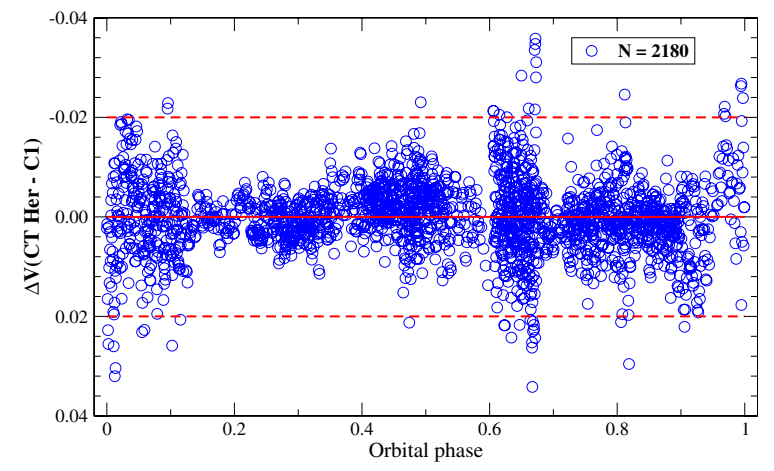

Fig. 4. Residual light curve phased against $P_{\text {orb }}($ filter $V$ ).

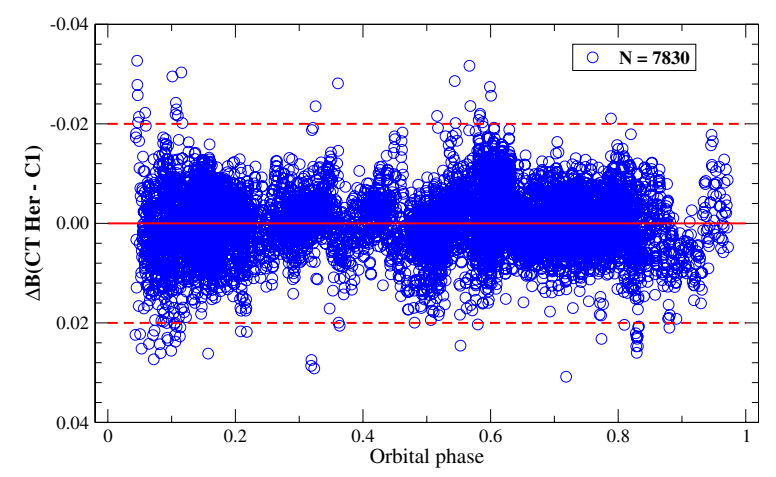

Fig. 5. Residual light curve phased against $P_{\text {orb }}($ filter $B)$.

eclipse of 2008 shows normal residuals. Such small effects might also have been introduced by the airmass-dependent corrections (since more prominent at the beginning/end of the night). After the removal of a few outliers larger than or equal to 0.02 mag, the remaining standard deviations are $6.4 \mathrm{mmag}$ in the $V$-band (with 1958 residual data points) and $6.3 \mathrm{mmag}$ in the $B$-band (with 7625 residual data points).

\subsection{Results from the B-data}

We performed Fourier analyses with PeRIOD04 (Lenz \& Breger 2005). Figure 6 illustrates the frequency search in the range $0-80 \mathrm{~d}^{-1}$ for the larger residual data set ( $B$-band): the spectral window is shown in the top panel, while the following panels show the initial periodogram and the periodograms successively prewhitened of the strongest signal from each previous run. The last panel shows the residual periodogram with the mean noise level computed for frequency bins of width $5 \mathrm{~d}^{-1}$. We identified fifteen frequencies with an amplitude-to-noise ratio larger than or equal to 4.0 (the empirical criterion introduced by Breger et al. 1993). The frequencies, amplitudes, residual standard deviations, $S / N$ s and the removed fraction of the initial variance, $1-R=\left(\sigma_{\text {init }}^{2}-\sigma_{\text {res }}^{2}\right) / \sigma_{\text {init }}^{2}$, obtained from a multiparameter fit of the residuals to a solution with fifteen frequencies, are listed in Table 6. The errors in the frequencies and the amplitudes were computed using 120 Monte Carlo simulations of Period04. The $S / N$ s are based on the mean noise levels of the periodograms of the residuals. We note that eight frequencies are concentrated in the range $43.5-53.5 \mathrm{~d}^{-1}$. The main frequency of $52.93664 \mathrm{~d}^{-1}$ has a semi-amplitude of $3.3 \mathrm{mmag}$ in $B$. This frequency corresponds to a periodicity of $27.2 \mathrm{~min}$, and is the one found by Kim et al. (2004a). A few oEA stars have even shorter main pulsation periods, for instance, RZ Cas
Table 6. $B$-band frequency-analysis of the residuals (model 72).

\begin{tabular}{lccccc}
\hline \hline ID & $\begin{array}{c}\text { Frequency } \\
( \pm \text { error }) \\
\mathrm{d}^{-1}\end{array}$ & $\begin{array}{c}\text { Amplit. } \\
( \pm 0.1) \\
\mathrm{mmag}\end{array}$ & $\begin{array}{c}\sigma_{\text {res }} \\
\mathrm{mmag}\end{array}$ & $S / N$ & $1-R$ \\
\hline$F_{1}$ & $52.93664( \pm 0.8)$ & 3.3 & 5.9 & 12.7 & \\
$F_{2}$ & $0.97682($ fixed $)$ & 2.8 & 5.6 & 11.4 & \\
$F_{3}$ & $47.59996( \pm 2)$ & 1.8 & 5.4 & 7.2 & \\
$F_{4}$ & $49.20822( \pm 2)$ & 1.8 & 5.3 & 6.6 & \\
$F_{5}$ & $2.37738($ fixed $)$ & 2.0 & 5.1 & 8.1 & \\
$F_{6}$ & $45.69130( \pm 3)^{*}$ & 1.3 & 5.0 & 6.2 & \\
$F_{7}$ & $1.00032($ fixed) & 2.1 & 4.9 & 8.4 & \\
$F_{8}$ & $45.44028( \pm 2)^{*}$ & 1.4 & 4.8 & 6.8 & \\
$F_{9}$ & 3.44103 (fixed) & 1.1 & 4.7 & 4.3 & \\
$F_{10}$ & $53.23747( \pm 2)$ & 1.2 & 4.6 & 4.8 & \\
$F_{11}$ & $4.08614($ fixed $)$ & 1.1 & 4.5 & 4.4 & \\
$F_{12}$ & 0.61211 (fixed) & 1.2 & 4.5 & 4.7 & \\
$F_{13}$ & 4.16902 (fixed) & 1.1 & 4.4 & 4.3 & \\
$F_{14}$ & $43.76799( \pm 3)$ & 1.1 & 4.4 & 5.4 & \\
$F_{15}$ & $45.56395( \pm 4)^{*}$ & 1.0 & 4.3 & 4.6 & 0.53 \\
\hline & & & & & \\
\hline
\end{tabular}

Notes. * Possibly affected by the $1 \mathrm{~d}^{-1}$ aliasing.

$\left(f_{1}=64.1935 \mathrm{~d}^{-1}\right.$, Rodríguez et al. 2004) and AS Eri $\left(f_{1}=\right.$ $59.03116 \mathrm{~d}^{-1}$, Mkrtichian et al. 2004).

All seven frequencies in the range $f<5 \mathrm{~d}^{-1}$ are caused by small imperfections related to the reduction, extraction of the orbital variations, and statistical fluctuations in the data sets. Since they are irrelevant to our study, we kept them fixed during the Monte Carlo simulations. Some of the other eight frequencies found are affected by the $1 \mathrm{~d}^{-1}$ aliasing phenomenon, and are flagged accordingly in Table 6 . At this point, we conclude that eight (pulsation) frequencies are found to be significant. After the prewhitening by the multi-frequency solution, the remaining standard deviation in the $B$-band equals $4.3 \mathrm{mmag}$, removing $53 \%$ of the initial variance. High-quality residual $B$-band light curves are presented in Fig. 7, that illustrate the good agreement between the residuals and the proposed solution.

\subsection{Results from the $V$-data}

Likewise, the complete set of the $V$-band residual data was analysed. Since these data are less numerous and more noisy, less significant results were found. We identified ten frequencies with amplitude-to-noise ratios larger than 4.0 , six of which are located in the range $43.5-53.5 \mathrm{~d}^{-1}$. The frequencies, amplitudes, residual standard deviations, $S / N \mathrm{~s}$, and the removed fraction of the initial variance, $1-R$, resulting from a multi-parameter fit to the $V$-band residual data set of a solution with ten frequencies, are listed in Table 7. All errors were computed using 120 Monte Carlo simulations of PERIOD04. Apart from the frequencies satisfying $f<5 \mathrm{~d}^{-1}$, the frequencies $G_{1}=F_{1}, G_{3}=F_{4}, G_{6}=F_{8}$, and $G_{9}=F_{14}$ were recovered, thus confirming the presence of at least four of the eight previously detected frequencies. The dominant frequency has a semi-amplitude of $2.2 \mathrm{mmag}$ and is located at $52.93762 \mathrm{~d}^{-1}$. It corresponds to the $(1050 \text { day })^{-1}$ alias frequency of $F_{1}$ produced by a three-year gap in the time series. In the $B$ band analysis, two unidentified frequencies $G_{4}$ (at $49.497 \mathrm{~d}^{-1}$ ) and $G_{10}\left(\right.$ at $\left.48.068 \mathrm{~d}^{-1}\right)$ were detected, whereas the frequency $F_{3}$ (at $47.600 \mathrm{~d}^{-1}$ ) was not confirmed. Whether these new frequencies might possibly be related to those already found, remains unclear. Furthermore, the frequencies $F_{6}$ and $F_{15}$, which form an almost equally spaced triplet with $G_{6}\left(=F_{8}\right)$, were also not recovered. In Sect. 5.3, we discuss these (apparently) partially 

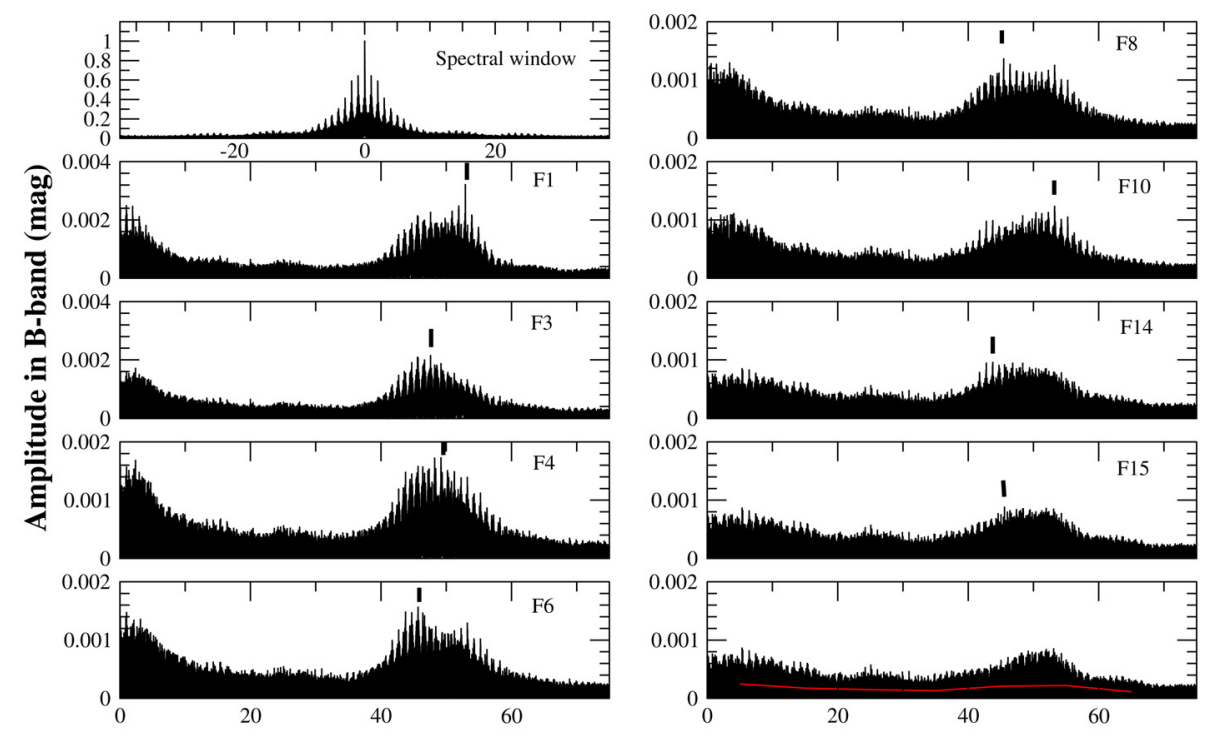

Frequency (cycles/day)
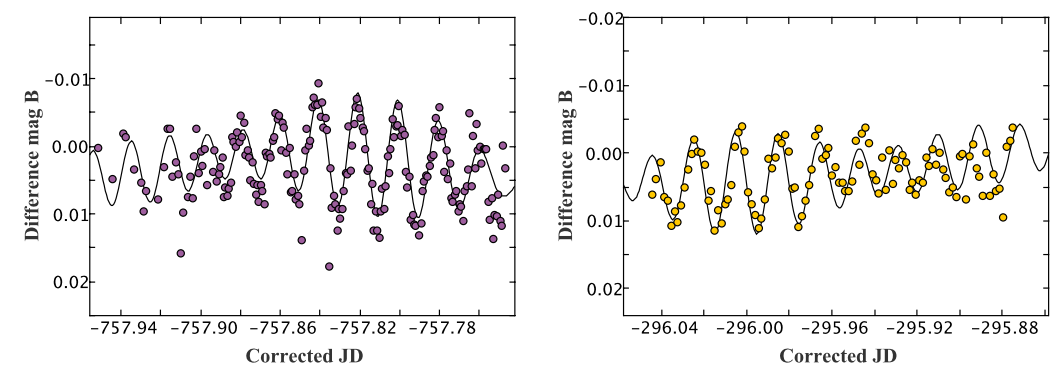

Fig. 6. Periodograms of CT Her computed during successive frequency analyses (filter $B$ ).
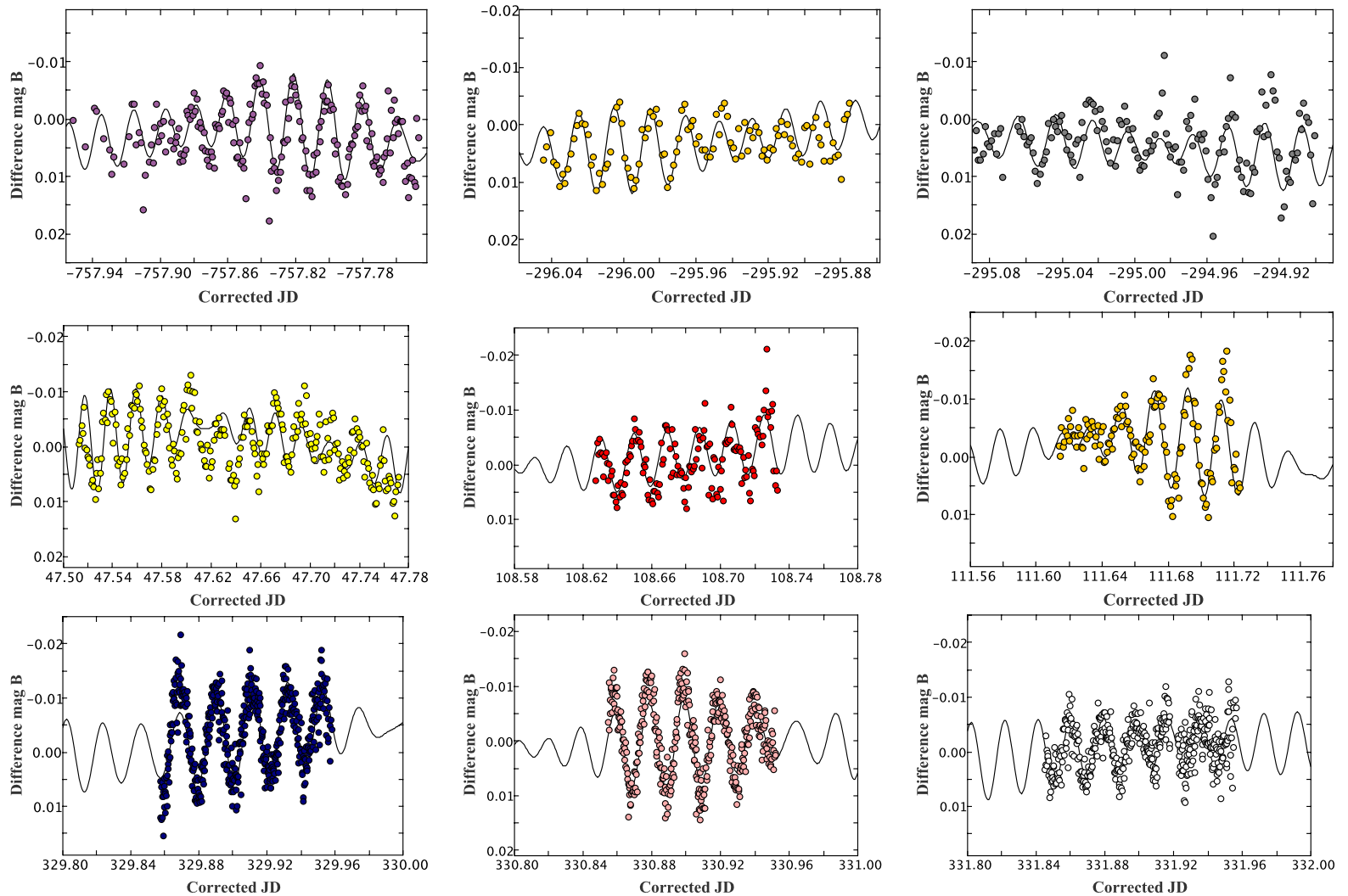

Fig. 7. Sample figures of the $B$-band residual data set and corresponding multi-frequency model.

incoherent results. After prewhitening by the solution with ten frequencies, the remaining standard deviation equals $5.1 \mathrm{mmag}$ in the $V$-band.

\subsection{Variability of the amplitudes of the intrinsic frequencies}

The full analyses in the $B$ and $V$-bands were repeated after subtraction of the alternative model using $T_{1}=8700 \mathrm{~K}$ (model 71). In the range above $24 \mathrm{~d}^{-1}$, the frequencies listed in Tables 6 and 7 were confirmed, though with slight modifications in the order of their appearance. This shows that the results of the Fourier analyses are perfectly independent of the adopted choice for the binary model owing to a) the binary models being almost identical and b) the different timescales involved.

It would also seem that the results of the frequency analyses in both filters are consistent with a common solution, were it not for the presence of $F_{3}$ (Table 6) and $G_{4}$ (Table 7). In addition, the order in which the frequencies were detected in the respective data sets is not identical. Therefore, we investigated whether patterns could be found in the pulsation amplitudes assuming that the frequency content is stable. All eight frequencies from Table 6 located in the range $43.5-53.5 \mathrm{~d}^{-1}$ were adopted to represent the frequency content of CT Her. Preference was given to the results from the $B$-band analysis because the aliasing is 
Table 7. $V$-band frequency-analysis of the residuals (model 72).

\begin{tabular}{cccccc}
\hline \hline ID & $\begin{array}{c}\text { Frequency } \\
( \pm \text { error }) \\
\mathrm{d}^{-1}\end{array}$ & $\begin{array}{c}\text { Amplit. } \\
( \pm 0.2) \\
\mathrm{mmag}\end{array}$ & $\begin{array}{c}\sigma_{\text {res }} \\
\mathrm{mmag}\end{array}$ & $S / N$ & $1-R$ \\
\hline$G_{1}$ & $52.93762( \pm 5)^{\&}$ & 2.2 & 6.4 & 7.4 & \\
$G_{2}$ & $0.56716($ fixed) & $2.2^{e}$ & 6.1 & 6.4 & \\
$G_{3}$ & $49.20636( \pm 6)^{\#}$ & 1.8 & 5.9 & 6.8 & \\
$G_{4}$ & $49.49661( \pm 7)$ & 1.6 & 5.6 & 6.2 & \\
$G_{5}$ & $3.68294($ fixed) & 1.9 & 5.5 & 5.6 & \\
$G_{6}$ & $45.43726( \pm 479)^{*}$ & 1.1 & 5.4 & 5.3 & \\
$G_{7}$ & $0.38478($ fixed) & 2.3 & 5.3 & 6.8 & \\
$G_{8}$ & $2.08342($ fixed) & 1.6 & 5.2 & 4.7 & \\
$G_{9}$ & $43.76795( \pm 22)$ & 1.1 & 5.3 & 5.4 & \\
$G_{10}$ & $48.06813( \pm 125)^{*}$ & 1.1 & 5.2 & 4.4 & 0.36 \\
\hline
\end{tabular}

Notes. * (Possibly) Affected by the $1 \mathrm{~d}^{-1}$ aliasing; \& is the $0.00095 \mathrm{~d}^{-1}$ alias frequency of $5.93664 \mathrm{~d}^{-1}\left(F_{1}\right.$, Table 6$) ;{ }^{\#}$ is the $0.0018 \mathrm{~d}^{-1}$ alias frequency of $49.20822 \mathrm{~d}^{-1}\left(F_{4}\right.$, Table 6$){ }^{e}$ the error is $\pm 0.3 \mathrm{mmag}$.

Table 8. Semi-amplitudes of the intrinsic frequenties ( $B$-band).

\begin{tabular}{|c|c|c|c|c|}
\hline ID & $\begin{array}{c}\text { Amplit. } \\
2004 \\
( \pm 0.3) \\
\text { mmag }\end{array}$ & $\begin{array}{l}\text { Amplit. } \\
2005 \\
( \pm 0.2) \\
\text { mmag }\end{array}$ & $\begin{array}{c}\text { Amplit } \\
2006 \\
( \pm 0.1) \\
\text { mmag }\end{array}$ & $\begin{array}{c}\text { Amplit. } \\
\text { 2007-2008 } \\
( \pm 0.1) \\
\text { mmag }\end{array}$ \\
\hline No. data & 951 & 1704 & 2262 & 2511 \\
\hline$\Delta T$ & 97 & 61 & 72 & 466 \\
\hline$F_{1}$ & 3.0 & 3.2 & 3.4 & 3.2 \\
\hline$F_{3}$ & $2.2( \pm 0.4)$ & 1.1 & $2.2^{e}$ & $1.6^{e}$ \\
\hline$F_{4}$ & 2.4 & 2.1 & 1.3 & $2.0^{e}$ \\
\hline$F_{6}$ & 2.2 & 1.7 & $0.7^{e}$ & 1.7 \\
\hline$F_{8}$ & 0.9 & 0.6 & $1.4^{e}$ & 2.2 \\
\hline$F_{10}$ & 1.8 & 1.2 & 1.2 & 1.8 \\
\hline$F_{14}$ & 0.2 & 1.0 & 1.5 & 0.8 \\
\hline$F_{15}$ & $1.3( \pm 0.5)$ & $0.5( \pm 0.5)$ & $1.2^{e}$ & $1.2^{e}$ \\
\hline
\end{tabular}

Notes. ${ }^{e}$ The error is $\pm 0.2 \mathrm{mmag}$.

much stronger in the $V$-band data (particularly the $1 \mathrm{~d}^{-1}$ and the $0.0018 \mathrm{~d}^{-1}$ aliasing effects). We then computed the multiparameter solutions with the amplitudes and the phases as free parameters on a yearly basis.

The results of the computations are shown in Table 8 and illustrated in Fig. 8. While the amplitude of the frequency $F_{1}$ can be considered to be stable over the entire observing season, this is not true for all the frequencies. The frequencies $F_{4}$ and $F_{6}$ behave similarly (first decreasing in amplitude until 2006 and then increasing), whereas the frequencies $F_{3}$ and $F_{8}$ show a maximum of their amplitude in 2006 and 2007, respectively. In contrast, the amplitudes of the frequencies $F_{14}$ and $F_{6}$ vary in anti-phase. This behaviour explains why the frequencies detected in Sects. 5.1 and 5.2 are not identical: the detection of $G_{1}=F_{1}, G_{3}=F_{4}$, and $G_{4}=F_{8}$ may be understood in the light of the 2005 (partially supplemented by the 2007-2008) $B$-band semi-amplitudes. In conclusion, the amplitudes of these pulsation frequencies display evidence of variability on timescales of 1-2 years, perhaps shorter, with the exception of the most dominant frequency, which has a stable amplitude.

\section{Radial velocities}

High-resolution spectra of CT Her were gathered with the 2-m telescope of the NAO, Rozhen, and the Coudé spectrograph equipped with an AT200 Photometrics camera (with a SITe SI003AB chip and pixel size of $24 \mu \mathrm{m}$ ), and a spectral

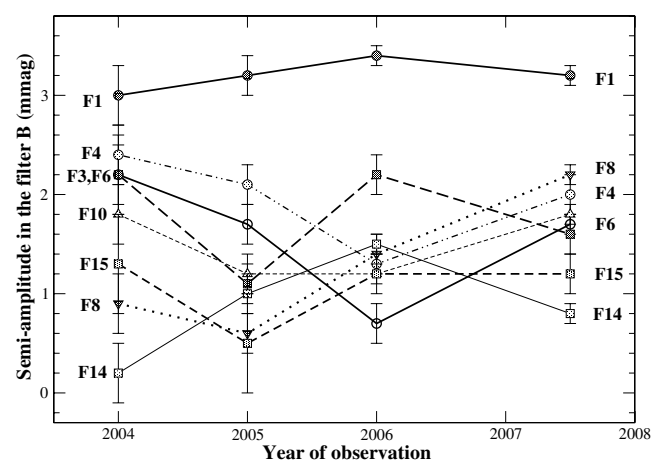

Fig. 8. Semi-amplitudes of the intrinsic frequencies versus time (filter $B$ ).
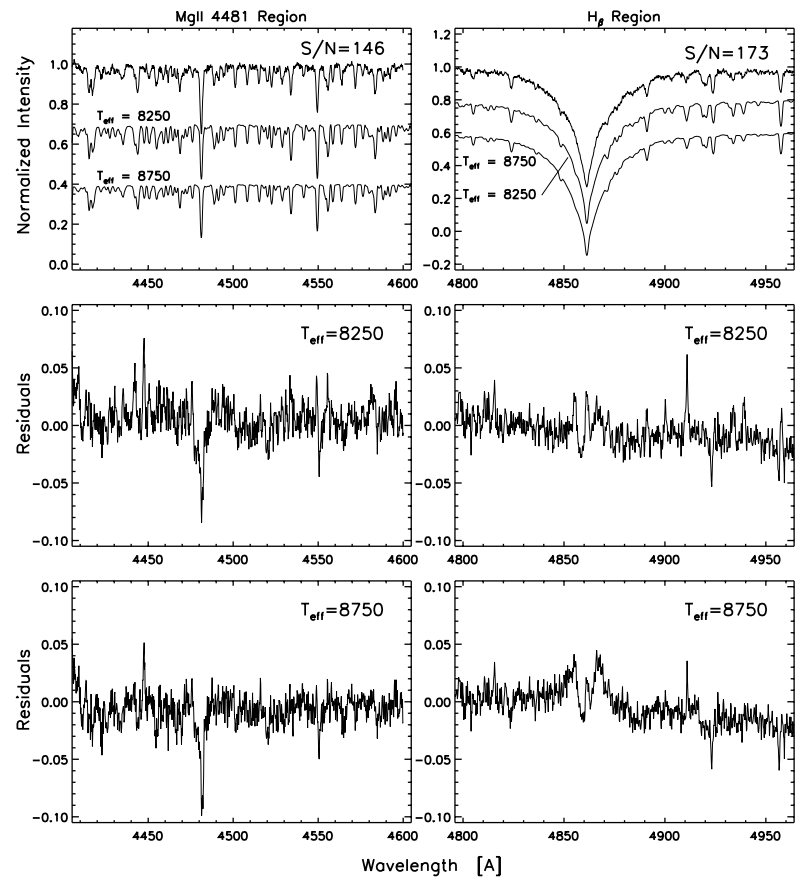

Fig. 9. Top panel: combined observational spectra and synthetic spectra showing model A $\left(T_{\text {eff }}=8250 \mathrm{~K}\right)$, and model B $\left(T_{\text {eff }}=8750 \mathrm{~K}\right)$. Other panels: difference spectra in the sense observed minus model A (middle) and observed minus model B (bottom).

resolution of $0.19 \AA /$ pixel. Three regions were observed: $\mathrm{H}_{\beta}$ and the regions around $\operatorname{MgII}(\lambda=4481 \AA)$ and FeI $(\lambda=5455 \AA)$. Forty-seven spectra were collected during 17 nights: 5 nights in May-July 2007, 5 nights in April-June 2008, as well as 7 nights in April-July 2009. Typical exposure times were 1200 and $1800 \mathrm{~s}$. The average $S / N$ is $\approx 30$. The spectra were reduced with standard IRAF procedures. The corresponding radial velocities were measured with the cross-correlation technique using synthetic spectra obtained with the programme SPECTRUM ${ }^{5}$ (Gray \& Corbally 2004), and a grid of LTE-atmosphere models of solar-type chemical composition (Castelli \& Kurucz 2003, Table 9). Combined spectra of $S / N>100$ were used to estimate the effective temperature of CT Her A: the best-fit function for two regions $\left(\mathrm{H}_{\beta}, \mathrm{MgII}\right)$ was obtained with $T_{\text {eff }}=8250 \mathrm{~K}$, $\log g=3.7$, and $v \sin i=60 \mathrm{~km} \mathrm{~s}^{-1}$ (Fig. 9).

Eight spectra were also collected with the Foces spectrograph attached to the DSAZ 2.2-m telescope of the Calar Alto Observatory (CLA) in 2009. The resolution is 40600 . The

5 http://www . phys. appstate.edu/spectrum/spectrum.html 

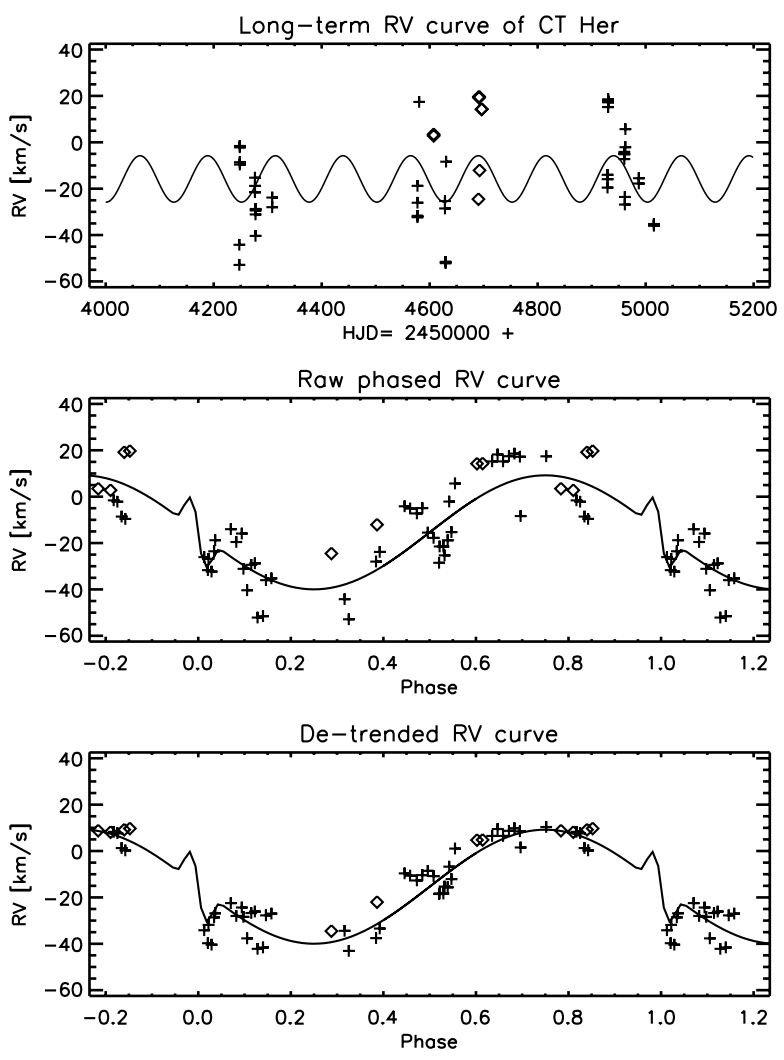

Fig. 10. Radial velocities of comp. A obtained from NAO spectra (plusses) and CLA spectra (diamonds): a 125.3 day periodicity was removed before fitting in combination with the $B$ - and $V$-light curves.

Table 9. Radial velocities of comp. A of CT Her.

\begin{tabular}{lcc}
\hline \hline HJD & RV & Error \\
\hline 2454247.47535 & -44.21 & 3.20 \\
2454247.49155 & -52.88 & 2.21 \\
2454248.37028 & -1.60 & 2.63 \\
$\ldots$ & & \\
\hline
\end{tabular}

Notes. First three lines, full table available at the CDS.

spectral region covers $5000-6000 \AA$. The average $S / N$ is $\approx 25$. The radial velocities of both components were measured with the cross-correlation technique using synthetic spectra generated for temperatures of $8200 \mathrm{~K}$ with $\log g=4.0$ and $4500 \mathrm{~K}$ with $\log g=3.5$, and for $v \sin i=50 \mathrm{~km} \mathrm{~s}^{-1}$. In this case, the two-dimensional cross-correlation programme ToDcor was used (Mazeh \& Zucker 1994). All radial velocity measurements are presented in Table 9, which is available at the CDS only. We first checked that the radial velocities obtained for component A were compatible with the NAO radial velocities. In a plot versus time (cf. top panel of Fig. 10), we detected a long-term variation that had to be removed before fitting.

A Fourier analysis of the residuals obtained from the raw velocities performed with PERIOD04, and a preliminary fit with velocity semi-amplitude $K_{\mathrm{A}}=23.4 \mathrm{~km} \mathrm{~s}^{-1}$ and systemic velocity $\gamma=-15.8 \mathrm{~km} \mathrm{~s}^{-1}$ indicated a periodicity of $\approx 125.3 \pm 2.2$ days with semi-amplitude $K_{\mathrm{C}}=11.4 \mathrm{~km} \mathrm{~s}^{-1}$. The corresponding ephemeris can be described by $\mathrm{HJD}_{\max }=2454157.6+$ $125.3 \times E$. Figure 10 shows the model (solid line in top panel) and the raw and corrected radial velocity curves (bottom panels). The standard deviations of the radial velocity residuals amount

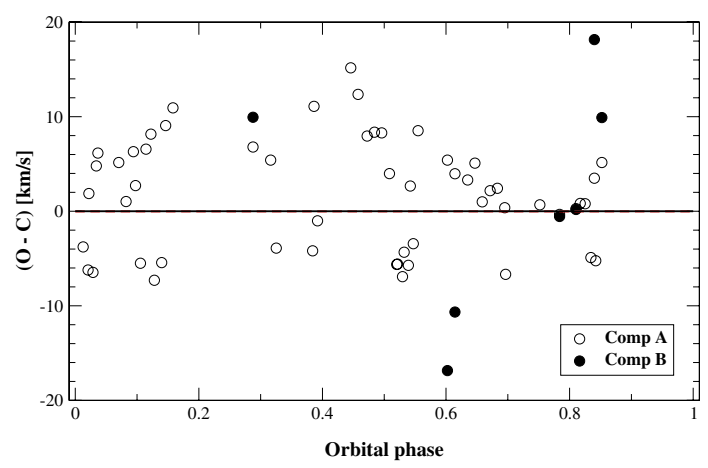

Fig. 11. Residual component radial velocities.

Table 10. Parameters of the combined light and radial velocity solution for CT Her.

\begin{tabular}{lcc}
\hline \hline Parameter & Filter $B$ & Filter $V$ \\
\hline$i\left(^{\circ}\right)$ & $82.75 \pm 0.04$ \\
$q$ & $0.1267 \pm 0.0006$ \\
$A$ & $8.48 \pm 0.03$ \\
$\gamma\left(\mathrm{km} \mathrm{s}^{-1}\right)$ & $-16.2 \pm 0.2$ \\
$T_{1}(\mathrm{~K})$ & \multicolumn{2}{c}{$8200^{a}$} \\
$T_{2}(\mathrm{~K})$ & $4468 \pm 7$ \\
$\Omega_{1}$ & $4.269 \pm 0.008$ \\
$\Omega_{2}{ }^{c}$ & \multicolumn{2}{c}{2.038} \\
{$\left[L_{1} /\left(L_{1}+L_{2}\right)\right]$} & 0.969 & 0.932 \\
{$\left[L_{2} /\left(L_{1}+L_{2}\right)\right]$} & 0.031 & 0.068 \\
$g_{1}$ & \multicolumn{2}{c}{$1.0^{a}$} \\
$g_{2}$ & \multicolumn{2}{c}{$0.32^{a}$} \\
$A_{1}$ & \multicolumn{2}{c}{$1.0^{a}$} \\
$A_{2}$ & \multicolumn{2}{c}{$0.5^{a}$} \\
$x_{1}{ }^{c}$ & $0.585^{b}$ & $0.505^{b}$ \\
$x_{2}{ }^{c}$ & $0.962^{b}$ & $0.811^{b}$ \\
\hline No. data & 7960 & 2180 \\
$\chi^{2}$ & 0.0162 & 0.0055 \\
No. RV data & $55($ comp A) & $7($ comp B $)$ \\
$\chi^{2}$ & 0.0038 & 0.0151 \\
\hline
\end{tabular}

Notes. ${ }^{(a)}$ Adopted value. ${ }^{(b)}$ Van Hamme (1993) tables. ${ }^{(c)} x_{1}, x_{2}$ are the limb darkening coefficients. $\Omega_{2}$ is the dimensionless potential of the secondary component.

to $11.69 \mathrm{~km} \mathrm{~s}^{-1}$ and $7.63 \mathrm{~km} \mathrm{~s}^{-1}$, respectively for the raw data and for the data after subtraction of the model.

The corrected radial velocity curves were next used in combination with the $B$ and $V$ light curves to perform fitting with Phoebe to determine the two parameters (1) the semi-axis major A (expressed in solar radii) and (2) the systemic velocity $\gamma$. The new parameter values and their formal uncertainties are listed in Table 10. This value of the semi-axis major lies close to its approximate value (Svechnikov \& Kuznetsova 1990). The values of the $\chi^{2}$ function are slightly larger than before. This new solution provides a slightly poorer fit to both light curves, particularly the primary eclipse in the $V$-filter, but accomodates the radial velocities reasonably: the means with their standard deviation of the residual radial velocities equals $1.7 \pm 5.8 \mathrm{~km} \mathrm{~s}^{-1}$ and $1.4 \pm 12.3 \mathrm{~km} \mathrm{~s}^{-1}$, for the components $\mathrm{A}$ and $\mathrm{B}$ respectively (Fig. 11). The main differences with the former solutions are the smaller value of the mass ratio $(q=0.127)$ and the higher inclination $\left(i=82.8^{\circ}\right)$. Table 11 summarizes the physical properties of both components resulting from the combined data fitting. These properties appear to be compatible with the status of component $\mathrm{A}$ as a pulsator of type $\delta$ Scuti. The computed masses are furthermore consistent with those preliminarily derived by 
Table 11. Tentative absolute parameters of CT Her.

\begin{tabular}{lcc}
\hline \hline Parameter & Comp. A & Comp. B \\
\hline Mass $\left(M_{\odot}\right)$ & $2.28 \pm 0.01$ & $0.29 \pm 0.04$ \\
Radius $\left(R_{\odot}\right)$ & $2.06 \pm 0.06$ & $1.87 \pm 0.08$ \\
$T_{\text {eff }}(\mathrm{K})$ & 8200 & $4468 \pm 80$ \\
$\log$ g & $4.17 \pm 0.02$ & $3.36 \pm 0.02$ \\
Luminosity $\left(L_{\odot}\right)$ & $17.4 \pm 2.4$ & $1.2 \pm 0.2$ \\
$M_{\text {bol }}(\mathrm{mag})$ & $1.65 \pm 0.15$ & $4.50 \pm 0.21$ \\
\hline
\end{tabular}

Hoffman \& Harrison (2009) from spectroscopy only: these authors obtained $M_{1}=2.31 \pm 0.11 M_{\odot}$ and $M_{2}=0.31 \pm 0.09 M_{\odot}$, with $q=0.13$ (in agreement with the $q$-values of Tables 5 and 10). We propose that this new solution illustrates the uncertainty still remaining in the characterization of the physical parameters of CT Her: more realistic errors would thus correspond to the formal errors of Table 5 (Table 10) multiplied with a factor of 25-50, while an uncertainty of about $80 \mathrm{~K}$ would be closer to the truth in the case of $T_{2}$. The data collected at the phase of primary minimum are probably affected by the pulsations (this is indeed the case for other oEA stars), which may introduce some uncertainty into the light-curve modelling. However, our radial velocity data, in particular of component $\mathrm{B}$, are too scarce to enable an accurate determination of the mass ratio. Accurate radial velocities for each component derived from high-resolution échelle spectra would be necessary to obtain a more consistent determination of the absolute parameters of CT Her. Meanwhile, the solution(s) derived in Sect. 4 are currently the most adequate one(s).

\section{Possible effect on the pulsation analysis}

The presence of a third body can cause a cyclic variation in the systemic velocity. In that case, the resulting light travel-time (LTT) effect will introduce periodical time delays in the pulsational analysis. We verified whether any phase shifts could be detected by subdividing the $B$-filter data into ten subsets arranged according to the orbital phase bin associated with the long-term ephemeris discussed in Sect. 6. We used the multivariate analysis method implemented in PERIOD04 (Breger 2005) to recompute the best-fit phases for each subset from the proposed multi-frequency solution. The outcome of these computations is that we find no obvious shift from the initially adopted phases. Figure 12 illustrates both the computed phase shifts, and that there is no detection of a systematic periodical pattern in the phases of the frequencies $F_{1}, F_{3}$, and $F_{4}$. It is clear that many data points in each subset are necessary to be able to reliably use the result of the multi-frequency fit, which is why we considered six out of ten subsets only. For these frequencies $\left(F_{1}, F_{3}\right.$ and $F_{4}$ ) and for these subsets, the errors in the phases are sufficiently precise.

Alternatively, one can derive a model that considers a periodic variation in the phase shifts of the multi-frequency solution. We also attempted this approach and the results confirm that no significant differences in the (pulsation) frequencies are detected even though a slightly tighter overall fit was obtained, corresponding to a 129.6 day LTT (solution with $\sigma_{\text {res }}=4.2 \mathrm{mmag}$ ).

\section{The $\mathrm{O}-\mathrm{C}$ diagram}

The same LTT effect also causes a cyclic variation in the orbital period. Therefore, we investigated the $\mathrm{O}-\mathrm{C}$ diagram of CT Her. We used the list of observed times of light minima from the

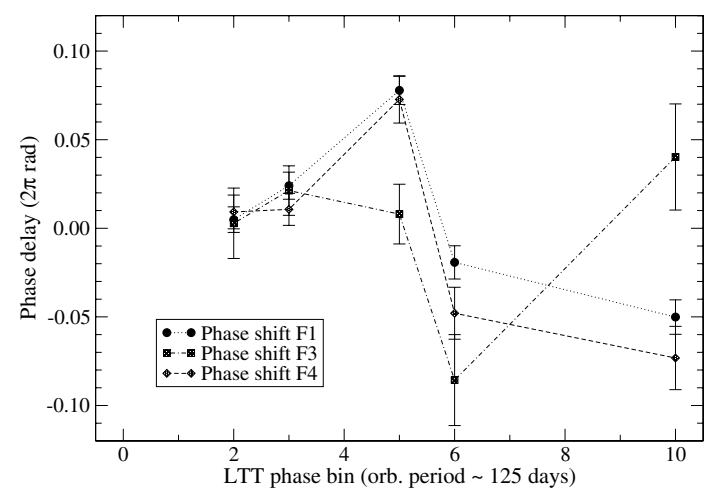

Fig. 12. Phase shifts computed for the pulsation frequencies $F_{1}, F_{3}$ and $F_{4}$ as a function of the orbital phase bin (with respect to the long periodicity).

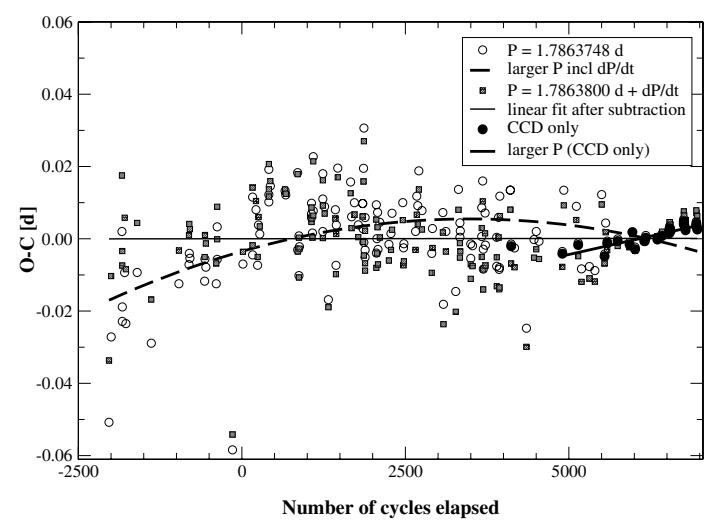

Fig. 13. Evolution of the orbital period of CT Her: O-C diagrams using a constant and a variable model.

database of the Variable Star and Exoplanet Section of the Czech Astronomical Society ${ }^{6}$, from which we selected 146 observed times collected between 1965 and 2009 (JD > 2438761 ), after elimination of the 26 first epochs. We then added two of our own observed minima: $2453189.3784 \pm 0.0002(E=5971)$ and $2453514.4961 \pm 0.0001(E=6153)$ (another minimum was not useful for the modelling of the $B$-band light curve because of an unexplained sudden drop during the eclipse and yet another (isolated) minimum was obtained by D. Litvinenko in an unreported filter). Using $P_{\text {orb }}=1.7863748$ days (Samus et al. 2009), we recomputed the $\mathrm{O}-\mathrm{C}$ values and searched for the best representation using either a linear or a parabolic model. Figure 13 illustrates both cases: the circles show the $\mathrm{O}-\mathrm{C}$ values with the known orbital period, while the squares show the $\mathrm{O}-\mathrm{C}$ values using the ephemeris corresponding to the best-fit parabola

$$
\begin{aligned}
\mathrm{HJD}_{\min }= & 2442522.92914+E \times 1^{\mathrm{d}} .7863799 \\
& -E^{2} \times 7^{\mathrm{d}} .394 \times 10^{-10} .
\end{aligned}
$$

This model infers a longer orbital period with a decline rate $\mathrm{d} P / \mathrm{d} t$ of $8.28 \times 10^{-10}$ days $\mathrm{d}^{-1}$ (i.e. a secular period decrease $\mathrm{d} P /(P \mathrm{~d} t)$ of $\left.169 \times 10^{-9} \mathrm{yr}^{-1}\right)$. The rms is 0.01049 days (906 s). To check its significance with respect to the linear model, we used an upper one-tailed F-test to verify whether the null hypothesis that the sums of squared residuals of both models are equal holds. The test statistic equals

$\left(\left(\chi_{\text {lin }}^{2}-\chi_{\text {par }}^{2}\right) \times(n-3)\right) /\left(\chi_{\text {par }}^{2} \times(3-2)\right)=23.44$

${ }^{6}$ O-C Gateway, http://var . astro.cz/ocgate/ocgate.php 
Table 12. O-C values for two models representing the evolution of $P_{\text {orb }}$.

\begin{tabular}{lccccc}
\hline \hline HJD & $\mathrm{E}$ & $\mathrm{O}-\mathrm{C}_{1}$ & $\mathrm{O}-\mathrm{C}_{2}$ & $\mathrm{p} / \mathrm{s}$ & Tech. \\
\hline$\ldots$ & & & & & \\
2438894.7540 & -2031 & -0.0508 & -0.0337 & $\mathrm{p}$ & vis \\
2438953.7280 & -1998 & -0.0271 & -0.0103 & $\mathrm{p}$ & vis \\
2439248.5090 & -1833 & 0.0020 & 0.0175 & $\mathrm{p}$ & vis \\
$\ldots$ & & & & & \\
\hline
\end{tabular}

Notes. First three used lines, full table available at the CDS.

Since this value is much larger than the tabulated value $F_{0.01}(1,145)=6.81$ (Pezzullo 2011), we reject the null hypothesis at the $1 \%$ significance level (type I error), meaning that the parabolic model is statistically significant. We note that Soydugan et al. (2008) used all previous O-C data (including the 26 first measurements) to derive an overall increase with a cyclic variation for the orbital period of CT Her. For evident reasons, we prefer to discuss only the well-observed part of this diagram.

Table 12 lists the cycle numbers and the associated residual values for both models. However, the first reported times do not fit these models at all as is also obvious from the $\mathrm{O}-\mathrm{C}$ diagram in the Atlas of $O-C$ diagrams of Eclipsing Binary Stars (Kreiner et al. 2001). Moreover, the scatter remains large in both cases. Interestingly, if we restrict the observations to only the 24 most recent times observed using the CCD technique, we obtain a tight-fitting linear model with a high correlation coefficient (0.9) indicating an increased orbital period of 1.7863789 days with respect to the known period (cf. the speckled circles in Fig. 13). The rms in this case is only 0.00135 days (117 s).

Using the information about a probable variation in systemic velocity with a period of 125.3 days and a semi-amplitude of $11.4 \mathrm{~km} \mathrm{~s}^{-1}$ (Sect. 6), we estimate a mass function equal to

$$
f(M)=1.0385 \times 10^{-7} \times\left(1-\mathrm{e}^{2}\right)^{3 / 2} \times K_{\mathrm{C}}^{3} P=0.019 M_{\odot}
$$

and a corresponding $\mathrm{O}-\mathrm{C}$ amplitude of $131 \mathrm{~s}$. Because this is of the same order as the computed rms (117 s), we conclude that the LTT effect is barely observable from the currently available data and that the O-C diagram of CT Her does not contradict the proposed model of a variable systemic velocity caused by the presence of a third body. We also note that the first two epochs of light minimum observed with a CCD display a flat trend, in full agreement with the period mentioned in the literature.

\section{Summary and conclusions}

The present study of the binary system CT Her is only the second of its kind for an oEA star (after the pioneering study of Y Cam), in which a large set of pulsation frequencies has been identified thanks to a long-term photometric monitoring performed in two filters. We have confirmed the presence of rapid pulsations with a dominant frequency of $52.93664 \mathrm{~d}^{-1}$. As many as eight significant frequencies, all within the range 43.5-53.5 $\mathrm{d}^{-1}$, appear to reproduce the observed, complex behaviour reasonably well throughout the years 2004-2008.

Furthermore, except for the most dominant frequency, we have shown that the amplitudes of these pulsation frequencies are variable on timescales of one to two years, perhaps even shorter than one year, assuming that the frequency content is stable (that several frequencies are commonly found in different data sets suggests this). On the basis of the absolute parameters of the (actual) primary component of CT Her (Table 11), we conclude that the dominant frequency cannot correspond to the fundamental radial mode but that instead such a short period of pulsation for a $2.5 M_{\odot}$ star is typical of a high overtone radial mode or a non-radial pulsation mode of high order "p" (similar to RZ Cas, AS Eri, or TW Dra). Using the improved ephemeris, we have derived an accurate value of 94.56 for the ratio $P_{\text {orb }} / P_{\text {puls }}$.

The analysis of recent spectra providing complementary radial velocities suggests that the light and radial velocity curves can be modelled simultaneously, provided that the radial velocity measurements are corrected for a long periodicity of $\approx 125$ days with a semi-amplitude $K_{\mathrm{C}}=11.4 \mathrm{~km} \mathrm{~s}^{-1}$. The presence of a third body in the system could explain such a light travel-time effect. However, we were unable to confirm this suggestion by analysing the O-C diagram of CT Her as the expected O-C amplitude is of the same order as that of the noise. We have been similarly unable to detect an obvious orbital modulation in the phases of the multi-frequency solution.

Although the match between the model and the $B$ - and $V$ light curves is excellent, we cannot yet claim that we have constrained the system parameters of CT Her uniquely, especially because accurate component radial velocities are currently unavailable. Such component radial velocities are needed in order to obtain a consistent determination of the absolute parameters of this Algol-type binary. Meanwhile, we acquired a new series of high-resolution spectra of CT Her with the Hermes spectrograph attached to the Mercator telescope (Raskin et al. 2011). High $S / N$ spectra should also be very useful to recover the components' spectra from the composite ones by applying spectra disentangling (e.g. TW Dra, Lehmann et al. 2009), allowing us to study the residual line profile variations and to spectroscopically identify the excited modes. The outcome from this study shows that CT Her is a particularly interesting system. The oEA stars indeed provide extremely useful asteroseismic targets because they offer the opportunity to study in detail the connection between pulsation and mass transfer in close binary systems, including the effects of tidal interaction.

Acknowledgements. Financial support from the Belgian Science Policy and the Bulgarian Academy of Sciences through the bilateral project "Astrometric, spectroscopic, and photometric follow-up of binary systems" (Ref. BL/33/B11) is gratefully acknowledged. A.S. thanks Prof. Y. Papamastorakis and Dr. I. Papadakis for the telescope time allocated at the Observatory of Skinakas. The data collected at BHO made use of equipment partially funded by the Belgian National Lottery (1999). This research made use of the SIMBAD and VIZIER databases, operated at CDS, Strasbourg, France, as well as of the ADS bibliography. We also thank the referee for most valuable comments.

\section{References}

Bessell, M. S. 1995, CCD Astronomy 2, No. 4, 20

Bíró, I. B., \& Nuspl, J. 2005, in Tidal Evolution and Oscillations in Binary Stars, ed. A. Claret, A. Giménez, \& J.-P. Zahn (San Francisco: ASP), ASP Conf. Ser., 333, 221

Breger, M., Stich, J., Garrido, R., et al. 1993, A\&A, 271, 482

Breger, M., et al. 2005, in The Light-Time Effect in Astrophysics, Causes and Cures of the O-C diagram, ed. C. Sterken (San Francisco: ASP), ASP Conf. Ser., 335,85

Castelli, F., \& Kurucz, R. 2003, in IAU Symp. 210, ed. N. Piskunov, W. W. Weiss, \& D. F. Gray (San Francisco: ASP), A20

De Cat, P., \& Aerts, C. 2002, A\&A, 393, 965

De Grève, J.-P., Mennekens, N., Van Rensbergen, W., \& Yungelson, L. R. 2009, in The 8th Pacific Rim Conference on Stellar Astrophysics, ed. B. Soonthornthum, S. Komonjinda, K. S. Cheng, \& K. C. Leung (San Francisco: ASP), ASP Conf. Ser., 404, 204

Dimitrov, D., Kraicheva, Z., \& Popov, V. 2008a, IBVS, 5842, 1

Dimitrov, D., Kraicheva, Z., \& Popov, V. 2008b, IBVS, 5856, 1

Dimitrov, D., Kraicheva, Z., \& Popov, V. 2009a, IBVS, 5883, 1 
Dimitrov, D., Kraicheva, Z., \& Popov, V. 2009b, IBVS, 5892, 1

Fitch, W. S. 1976, in Multiple Periodic Variable Stars, Budapest, Hungary, ed. W. S. Fitch (D. Reidel Publishers), IAU Coll., 29, 167

Gamarova, A. Yu., Mkrtichian, D. E., Rodríguez, E., et al. 2003, in Interplay of Periodic, Cyclic and Stochastic Variability in Selected Areas of the H-R Diagram, ed. C. Sterken (San Francisco: ASP), ASP Conf. Ser., 292, 369

Gray, R., \& Corbally, C. 2004, AJ, 207, 742

Handler, G., Balona, L. A., Shobbrook, R. R., et al. 2002, MNRAS, 333, 262

Hoffman, D. I., \& Harrison, T. E. 2009, in Stellar Pulsation: Challenges for Theory and Observation, ed. J. A. Guzik, \& P. A. Bradley (NY: Melville), AIP Conf. Proc., 1170, 429

Kim, S.-L., Koo, J.-R., Lee, J. A., et al. 2004a, IBVS, 5537, 1

Kim, S.-L., Lee, J. W., Kwon, S.-G., et al. 2004b, A\&A, 405, 231

Kim, S.-L., Kim, S. H., Lee, D.-J., et al. 2005, in Tidal Evolution and Oscillations in Binary Stars, ed. A. Claret, A. Giménez, \& J.-P. Zahn (San Francisco: ASP), ASP Conf. Ser., 333, 217

Kim, S.-L., Lee, C.-U., \& Lee, J. W. 2006, Mem. Soc. Astron. It., 77, 184

Kreiner, J. M., Kim, C.-H., \& Nha, I.-S. 2001, An Atlas of O-C diagrams of Eclipsing Binary Stars, Cracow, Poland: Wydawnictwo Naukowe Akademii Pedagogicznej, http://www .as .ap.krakow.pl/o-c

Kurtz, D. W. 2000, in Delta Scuti and Related Stars, ed. M. Breger, \& M. Montgomery (San Francisco: ASP), ASP Conf. Ser., 210, 287

Kuznetsova, Eh. F., \& Svechnikov, M. A. 1990, TarOT, 107, 76

Lampens, P. 2006, in Astrophysics of Variable Stars, ed. C. Sterken, \& C. Aerts (San Francisco: ASP), ASP Conf. Ser., 349, 153

Lampens, P., Strigachev, A., Kim, S.-L., et al. 2008a, Commun. Asteroseism., 153,54

Lampens, P., Strigachev, A., Kim, S.-L., et al. 2008b, Commun. Asteroseism., 157,328

Landolt, A. U. 1992, AJ, 104, 340

Lehmann, H., \& Mkrtichian, D. 2004, A\&A, 413, 293

Lehmann, H., \& Mkrtichian, D. 2008, A\&A, 480, 247

Lehmann, H., Tkachenko, A., \& Mkrtichian, D. E. 2009, JENAM 2008 Symp. 4: Asteroseismology and Stellar Evolution, ed. S. Schuh, \& G. Handler, Commun. Asteroseism., 159, 45

Lenz, P., \& Breger, M. 2005, CoAst, 146, 53

Maceroni, C. 2006, in Astrophysics of Variable Stars, ed. C. Sterken, \& C. Aerts (San Francisco: ASP), ASP Conf. Ser., 349, 41

Mason, B. D., Gies, D. R., \& Hartkopf, W. I. 2001, in The influence of binaries on stellar population studies, ed. D. Vanbeveren (Dordrecht: Kluwer Academic Publishers), ASSL, 264, 45

Mayor, M., Udry, S., Halbwachs, J.-L., et al. 2001, in The Formation of Binary Stars, ed. H. Zinnecker, \& R. D. Mathieu (San Francisco: ASP), IAU Symp., 200,47
Mazeh, T., \& Zucker, S. 1994, Ap\&SS, 212, 349

Michalska, G., \& Pigulski, A. 2008, J. Phys. Conf. Ser., 118, 2064

Mkrtichian, D. E., Kusakin, A. V., Gamarova, A. Yu., et al. 2002, in Observational Aspects of Pulsating B- and A Stars, ed. C. Sterken, \& D. W. Kurtz (San Francisco: ASP), ASP Conf. Ser., 256, 259

Mkrtichian, D. E., Kusakin, A. V., Rodríguez, E., et al. 2004, A\&A, 419, 1015

Mkrtichian, D. E., Rodríguez, E., Olson, E. C., et al. 2005, in Tidal Evolution and Oscillations in Binary Stars, ed. A. Claret, A. Giménez, \& J.-P. Zahn (San Francisco: ASP), ASP Conf. Ser., 333, 197

Mkrtichian, D., Kim, S.-L., Kusakin, A. V., et al. 2006, Ap\&SS, 304, 169

Mkrtichian, D. E., Kim, S.-L., Rodríguez, E., et al. 2007, in Solar and Stellar Physics Through Eclipses, ed. O. Demircan, S. O. Selam, \& B. Albayrak (San Francisco: ASP), ASP Conf. Ser., 370, 194

Papadakis, I., Boumis, P., Samaritakis, V., et al. 2003, A\&A, 397, 565

Pezzullo, J. C. 2011, Interactive Statistics Pages, http://statpages.org/pdfs.html

Pigulski, A., \& Michalska, G. 2007, Acta Astron., 57, 61

Prša, A., \& Zwitter, T. 2005a, Ap\&SS, 296, 315

Prša, A., \& Zwitter, T. 2005b, ApJ, 628, 426

Raskin, G., Van Winckel, H., Hensberge, H., et al. 2011, A\&A, 526, A69

Reed, M. D., \& Brondel, B. J. 2005, in Tidal Evolution and Oscillations in Binary Stars, ed. A. Claret, A. Giménez, \& J.-P. Zahn (San Francisco: ASP), ASP Conf. Ser., 333, 228

Rodríguez, E., García, J. M., Mkrtichian, D. E., et al. 2004, MNRAS, 347, 1317

Rodríguez, E., García, J. M., Costa, V., et al. 2010, MNRAS, 408, 2149

Rucínski, S. M. 1969, Acta Astron., 19, 245

Samus, N. N., Durlevich, O. V., et al. 2009, Combined General Catalogue of Variable Stars, VizieR Online Data Catalog (GCVS4.2, version 2009Mar)

Soydugan, F., Soydugan, E., Ibanoglu, C., et al. 2006, AN, 327, 905

Soydugan, F., Kacar, Y., Soydugan, E., et al. 2008, Commun. Asteroseism., 157, 321

Stetson, P. 1987, PASP, 99, 191

Strigachev, A. 2009, Bulg. Astron. J., 11, 87

Svechnikov, M. A., \& Kuznetsova, Eh. F. 1990, Catalogue of approximate photometric and absolute elements of eclipsing variable stars, VizieR Online Data Catalog (V/124)

Tkachenko, A., Lehmann, H., \& Mkrtichian, D. 2010, AJ, 139, 1327

Van Hamme, W. 1993, AJ, 106, 2096

Wilson, R. E. 1979, ApJ, 234, 1054

Wilson, R. E. 1990, ApJ, 356, 613

Wilson, R. E., \& Devinney, E. J. 1971, ApJ, 166, 605

Witte, M. G., \& Savonije, G. J. 1999, A\&A, 350, 129 